\title{
FliC-delta220-320 from Salmonella enteritidis as an Adjuvant for Vaccine Development
}

\author{
Bao-Chau Thi Tran ${ }^{1,2}$, Hai-Vy Vo-Nguyen ${ }^{1,2}$, Viet-Anh Nguyen ${ }^{1,2}$, Thuoc Linh Tran ${ }^{1,2}$, Hieu Tran-Van ${ }^{1,2 *}$ \\ ${ }^{1}$ Department of Molecular and Environmental Biotechnology, Faculty of Biology and Biotechnology, University of \\ Science, Ho Chi Minh City, Vietnam \\ ${ }^{2}$ Vietnam National University, Ho Chi Minh City, Vietnam
}

\begin{abstract}
*Address for Correspondence: Dr. Hieu Tran-Van, Associate Professor, Faculty of Biology and Biotechnology, HCMC University of Science, Vietnam National University, HCM City, Vietnam

E-mail: tvhieu@hcmus.edu.vn ; Fax Number: +842838350096
\end{abstract}

Received: 25 Sep 2019/ Revised: 28 Dec 2019/ Accepted: 29 Feb 2020

\begin{abstract}
Background: High efficacy of subunit vaccine requires immunostimulant as an adjuvant component. Flagellin protein (FliC) in bacteria is considered as a potential candidate. However, the high antigenic property of FliC has made it difficult to apply for a variety of vaccine development. In this present study, we assessed the immunostimulant of Flic ${ }_{\Delta 220-320^{-}}$a less immunogeneicity variant of FliC. In which, 100 amino acids were deleted from the hypervariable domain of FliC in fusion with GFP (plays as a model antigen).

Methods: To generate this contract, we isolated FliC gene from Salmonella enterica Enteritidis (S. enteritidis) serving for cloning and expressing of FliC-delta220-320 ( $F l i C \Delta_{220-320}$-gfp) protein in E. coli. The gfp gene was cloned into pET28a-FliC $\mathrm{C}_{\Delta 220-320}$ resulting in a recombinant vector $\mathrm{pET} 28 \mathrm{a}-\mathrm{FliC}_{\Delta 220-320}$-gfp. The expression of FliC $\Delta_{220-320}$-gfp protein was induced by $0.1 \mathrm{mM}$ IPTG and confirmed by the SDS-PAGE analysis and Western blot probed with anti-6xHis antibody. This recombinant protein was then validated for immunostimulatory via subcutaneous immunization in mice.

Results: FliC $_{\Delta 220-320-g f p}$ protein was expressed in soluble form and was purified by using the immobilized-metal affinity chromatography with a purity of $56 \%$. The present results showed that the $\mathrm{FliC}_{\triangle 220-320}$ variant in fusing with GFP reduced its antigenic stimulation to four times than that of FliC in the FliC-gfp and retained its ability to stimulate a humoral immune response against fused GFP.

Conclusion: This result suggested that it was possible to apply the FliC ${ }_{\Delta 220-320}$ variant to subunit vaccines in a form of fusion with protein antigen(s) to increase their efficacy.
\end{abstract}

Key-words: FliC $_{\Delta 220-320,}$ Hypervariable domain, Immunoadjuvants, Salmonella enterica Enteritidis, Subunit vaccine

\section{INTRODUCTION}

Infectious diseases remain the second leading cause of death worldwide, but the first in infants and children ${ }^{[1]}$. Vaccination is one of the most successful public health interventions ever undertaken and continues to have a tremendous impact in preventing the spread and death from infectious diseases around the world by generating a pathogen-specific immune response with a long-lasting protection ${ }^{[2]}$.

\section{How to cite this article}

Tran BCT, Vo-Nguyen HV, Nguyen VA, Tran TL, Tran-Van H. FliCdelta220-320 from Salmonella enteritidis as an Adjuvant for Vaccine Development. SSR Inst. Int. J. Life Sci., 2020; 6(2): 24942501.

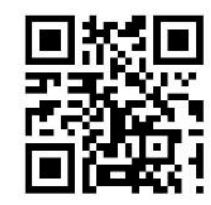

Live-attenuated vaccines, inactivated vaccines and subunit vaccines are the three different types of vaccine currently used and more popular with humans ${ }^{[3]}$. Liveattenuated vaccines are considered to be the most effective, but its side effect reduces its vaccine of choice. Inactivated vaccines get rid of reversion to virulence, but can only stimulate a humoral immune response with little or no cell-mediated response ${ }^{[4]}$. Subunit vaccines contain part of the pathogen that has been purified or synthesized. Although considered safe, subunit vaccine is not able to stimulate a strong immune response. This problem can be addressed by the addition of immune adjuvants, which helps boost immune responses against antigens or modulates it towards the desired immune responses ${ }^{[5]}$. In addition, other characteristics that need to be considered when selecting an immune adjuvant are 
types of activated immune response, vaccinated species, and routes of administration.

One of the constituents of flagella of Salmonella sp., FliC has long been recognized as a potential adjuvant due to its effective immune stimulation when interacting with cognate receptor on the surface of innate immune cells [6]. TLR5 was identified as an extracellular receptor of FliC [7]. When being fused to antigens, FliC favorably facilitates conditions for antigens to be presented by antigen-presenting cells due to its affinity for TLR5s on the APC surface ${ }^{[8,9]}$. However, FliC's antigenicity needs to be eliminated or reduced to be applied in various vaccines ${ }^{[10]}$. Many studies have implemented different methods to alter the FliC protein, such as muting or eliminating some parts of the protein sequences, to discover FliC variants with less antigenicity but still retain stimulating characteristics with TLR5. In particular, Liu et al. ${ }^{[11]}$ have shown that the removal of 100 amino acids in the FliC hypervariable domain created the $\mathrm{FliC}_{\Delta 220-320}$ variant with the ability to stimulate the production of proinflammatory cytokines, which is not only equivalent to the intact FliC, but also significantly reduces its antigenicity ${ }^{[11]}$. However, in their experiments, antigen was simply mixed with FliC $_{\Delta 220-320}$ but not covalently linked to the variant. This could reduce immune stimulatory of the FliC $_{\Delta 220-320}$. In this study, we evaluated the immune stimulatory of recombinant FliC $_{\Delta 220-320}$ biologically linked to GFP (plays as an antigen). The model could provide more evidences for the development of highly efficient recombinant vaccines.

\section{MATERIALS AND METHODS}

This study performed in March, 2019 at the Department of Molecular and Environmental Biotechnology, Faculty of Biology and Biotechnology, University of Science, VNU-HCM, Hochiminh City, Vietnam.

Bacterial strains, plasmids, reagents and growth conditions- E. coli $\mathrm{DH} 5 \alpha$ \{F- end A1 hsdR17 (rk-/mk-) supE44 thi $\lambda$-recA1 gyrA96 $\Delta$ lacU169 ( $\varphi 80$ lacZ $\Delta M 15)\}$ and $E$. coli BL21 (DE3) (F+ ompT hsdSB (rB- mB-) gal dcm (DE3) were used as host strains for cloning and protein expression, respectively. Both were routinely grown in Luria-Bertani (LB) medium containing $50 \mu \mathrm{g} / \mathrm{mL}$ of kanamycin at $37^{\circ} \mathrm{C}$. S. enterica Enteritidis and pET-gfp vector were used to obtain the gene encoding for flagellin protein and GFP protein, respectively. The pET28a plasmid was used as a vector for cloning the fusion genes $\mathrm{FliC}_{\Delta 220-320-\mathrm{gfp}}$ and FliC-gfp, and protein expression controlled by T7 promoter via the IPTG (Isopropyl ß-D-1-thiogalactopyranoside) (Biobasic) inducer. All strains and plasmids were provided by the Department of Molecular and Environmental Biotechnology, University of Natural Sciences, VNU-HCM, Vietnam. Recombinant proteins were constructed, expressed and stored at $-20^{\circ} \mathrm{C}$.

Construction of recombinant pET28a-FliC ${ }_{\Delta 220-320-g f p}$ and pET28a-FliC-gfp- GFP gene obtained from pET-gfp vector and pET28a-FliC ${ }_{\Delta 220-320}$ vector (unpublished results) were digested to create sticky ends with HindIII and Xhol (Thermo Scientific). The ligation reaction containing the double-digested insert and the expression vector, was performed in the presence of T4 DNA ligase (Thermo Scientific) and the resulting mixture was transformed into competent $E$. coli $\mathrm{DH} 5 \alpha$. The transformants were initially screened on kanamycin-containing LB agar plate, then re-screened by the PCR with specific primers (F: CATATGgcacaagtcattaatacaaacagcc and R: CTCGAGacgcagtaaagagaggacgttttgc) and T7pro/T7ter. Plasmids derived from positive colonies confirmed by PCR colonies were sent to the Phu Sa Biochemical Company for sequencing. The $E$. coli $\mathrm{DH} 5 \alpha$ strain carrying the target plasmid pET28a-FliC-gfp ${ }^{[12]}$ was created in a completely similar manner, but replaced pET28a-FliC ${ }_{\Delta 220-}$ 320 with pET28a-FliC (Fig. 1).

Expression of recombinant FliC $_{\Delta 220-320-g f p}$ and FliC-gfp in E. coli BL21 (DE3)- The expression of recombinant FliC $_{\Delta 220-320-g f p}$ and FliC-gfp were conducted as described with some modifications ${ }^{[13]}$. pET28a-FliC ${ }_{\Delta 220-320}$ and pET28a-FliC obtained from previous steps were transformed into competent E. coli BL21 (DE3) to create vector-carrying strains. Positive colonies were inoculated in LB media shaking tubes supplemented with kanamycin and allowed to grow at $37^{\circ} \mathrm{C}$ in 16 hours. The cultures were then sub-cultured at 1:10 (v/v) and inoculated at $37^{\circ} \mathrm{C}$ until $\mathrm{OD}_{600}$ reached $0.8-1.0$. At this point, the cultures were induced with $0.1 \mathrm{mM}$ concentration of IPTG and the protein expressions were performed at $16^{\circ} \mathrm{C}$ in 16 hours. Harvested cells proceeded for sonication on ice to obtain proteins in total, soluble and precipitated phases. Before the induction, a sample of the bacterial culture was taken as negative control with E. coli BL21 (DE3)/ pET28a induced by IPTG with the same concentration. 
Recombinant protein expressions were analyzed by SDSPAGE and Coomassie Brilliant Blue stained, followed by
Western blot and probed with anti-His-tag antibody (Santa Cruz).

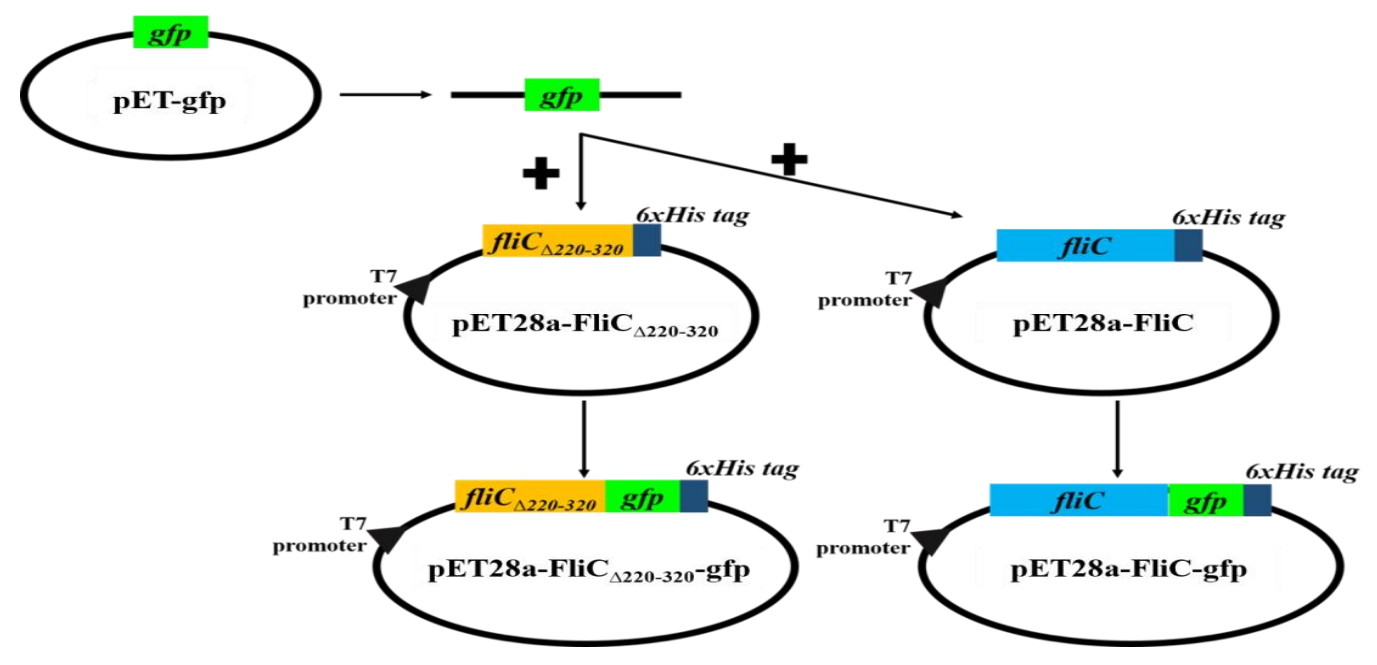

Fig. 1: Cloning strategies for $p E T 28 a-F l i C_{\Delta 220-320^{-}}$gfp and pET28a-FliC-gfp recombinant vectors

\section{SDS-PAGE and Western blotting- SDS-PAGE and Western} blotting were performed as described with some modifications ${ }^{[13]}$. Confirmation of the $\mathrm{FliC}_{\Delta 220-320-\mathrm{gfp}}$ and FliC-gfp expression was performed using $12.5 \%$ SDSPAGE gel, followed by Western blotting. The proteins from the gel were transferred to the nitrocellulose membrane and were probed with mouse-anti-His-tag antibody, then detected by rabbit-anti-mouse IgG-HRP (Santa Cruz).

Purification of recombinant FliC $_{\Delta 220-320-g f p}$ and FliC-gfpThe purification of recombinant $\mathrm{FliC}_{\triangle 220-320}$-gfp and FliCgfp were conducted as described with some modifications ${ }^{[13]}$. The supernatant of E. coli BL21 (DE3)/pET28a-FliC ${ }_{\Delta 220-320-g f p}$ (or pET28a-FliC-gfp) obtained from sonicated step was used as raw material to purify $\mathrm{FliC}_{\Delta 220-320-\mathrm{gfp}}$ (or FliC-gfp) by affinity chromatography with HP Hitrap column (GE Healthcare). After being equilibrated with solution A ( $50 \mathrm{mM}$ Tris- $\mathrm{HCl}$, $100 \mathrm{mM} \mathrm{NaCl}, 20 \mathrm{mM}$ imidazole $\mathrm{pH}$ 8.0), the column was loaded with soluble protein solution, then rebalanced and washed with solution A. Finally, the target protein was eluted with solution $B(50 \mathrm{mM}$ Tris $-\mathrm{HCl}, 100 \mathrm{mM}$ $\mathrm{NaCl}, 200 \mathrm{mM}$ imidazole, $\mathrm{pH}$ 8.0). Purified $\mathrm{FliC}_{\Delta 220-320-\mathrm{gfp}}$ (or FliC-gfp) was tested by the SDS-PAGE, Coomassie Brilliant Blue stained and analyzed by the Image J software. Recombinant GFP protein (pET-gfp) was also expressed, obtained and purified for the evaluation of

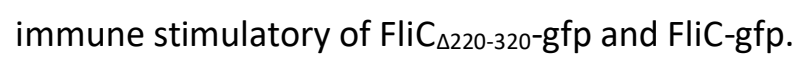

Immunizated mice with recombinant FliC $\mathrm{C}_{\Delta 220-320-\mathrm{gfp}}$ and Flic-gfp- Immunization of mice was conducted as described with some appropriate modifications ${ }^{[13]}$. Swiss mice (Mus musculus var. albino) male, 3-5 weeks old, healthy, average weight of $10-15 \mathrm{~g}$, provided by the HCMC Drug Testing Institute, were divided into 4 experimental groups $(n=5)$. (i) and (ii): positive control and negative control, respectively, injected with $25 \mathrm{ng}$ GFP/dose; (iii) Group 1, injected with $67 \mathrm{ng} \mathrm{FliC}_{\triangle 220-320^{-}}$ gfp/dose; (iv) Group 2, injected with 77 ng FliC-gfp/dose. FCA (Freund's Complete Adjuvant-Santa Cruz) was used for the first dose of positive control ( $50 \mu \mathrm{L} /$ dose), FIA (Freund's Incomplete Adjuvant-Santa Cruz) was used for the first dose of negative control, test groups and booster doses ( $50 \mu \mathrm{L} /$ dose). Booster dose was injected after three weeks, and then repeated every two weeks with half the amount of antigen, with the unchanged volume and percentage of adjuvants. The amount of protein used for injection was calculated based on the amount of target protein after purification and equivalent to $25 \mathrm{ng}$ GFP. One week after the last injection, 200-300 $\mu \mathrm{L}$ of blood was obtained from the orbital vein of each mouse; serum was collected and stored at $-20^{\circ} \mathrm{C}$ for later analysis.

Evaluation of immune stimulatory of recombinant FliC $_{\Delta 220-320-g f p}$ and FliC-gfp by indirect ELISA- Specific antibody titers were determined by indirect ELISA as described with some modifcation [13]. The 96-well microtiter plates were coated with $2 \mu \mathrm{g} / \mathrm{mL}$ antigen (GFP or FliC) in $100 \mu \mathrm{L}$ carbonate buffer $(\mathrm{pH} \mathrm{9.6)}$ at room 
temperature in 2 hours and then blocked with $100 \mu \mathrm{L}$ of 5\% skim milk in PBST for 1 hour at room temperature. After being washed three times with PBST, $100 \mu \mathrm{L}$ of mouse-anti-serum, diluted in 2-fold dilution manner from 1:5,000 to 1:640,000, were added and incubated at room temperature for 1 hour. Solutions were removed and $100 \mu \mathrm{L} /$ well of rabbit-anti-mouse IgG-HRP at 1:10,000 dilution was added and incubated for 1 hour at room temperature. The plates were developed using 100 $\mu \mathrm{L}$ of 3,3 ', 5, 5'-Tetra methyl benzidine (TMB). The reaction was stopped with $100 \mu \mathrm{L}$ of $\mathrm{H}_{2} \mathrm{SO}_{4} 2 \mathrm{~N}$ after 30 minutes, and the absorbance at $450 \mathrm{~nm}$ was measured on a microplate reader (Thermo Electron Corporation). Negative control was created in a completely similar manner, but replaced with the serum sample collected before the first dose.

\section{RESULTS}

Molecular cloning of recombinant pET28a-FliC ${ }_{\Delta 220-320-g f p}$ and PET28a-FliC-gfp- PCR colonies were performed to screen for recombinant pET28a-FliC ${ }_{\Delta 220-320-g f p}$ and pET28a-FliC-gfp from transformed E. coli DH5a. Amplicons with sizes of approximately $1215 \mathrm{bp}$ and 1515 bp were obtained by using specific primers for $\mathrm{FliC}_{\Delta 220-320}$ and FliC ( $F$ and R), respectively (lane 2 and 4, Fig. 2). When using T7pro/ T7ter primers, the resulted bands were approximately 2230 bp and 2530 bp (lane 3 and 5, Fig. 2), including the $\mathrm{FliC}_{\triangle 220-320}$ gene in case of $\mathrm{pET} 28 \mathrm{a}$ -

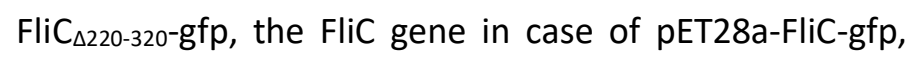
respectively, and the gfp gene incorporated with the length from T7 pro/ter to the target genes. The recombinant vectors of desire were confirmed.

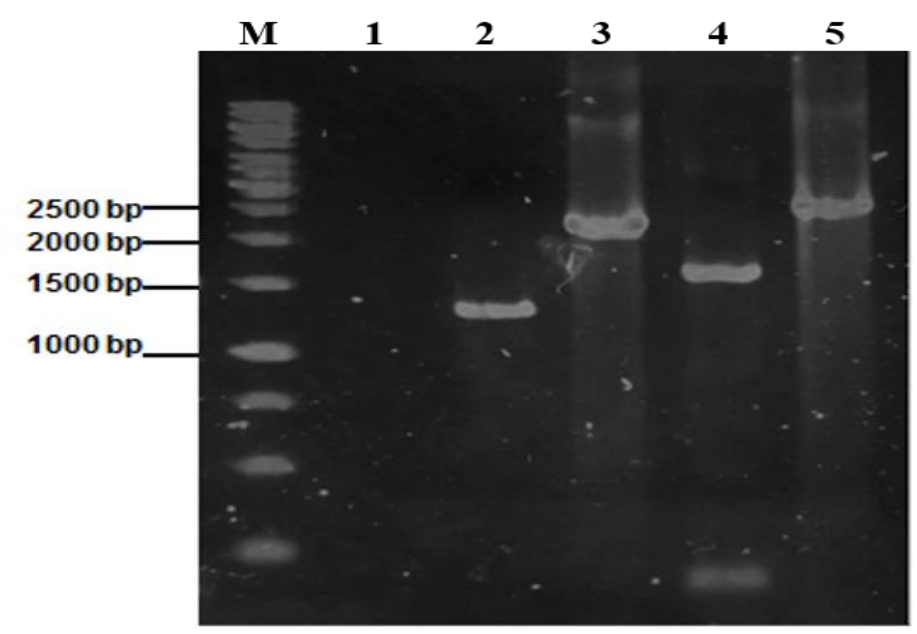

Fig. 2: PCR colonies of $E$. coli (DH5 $\alpha) / p E T 28 a-F_{1 i C}{ }_{\triangle 220-320^{-}}$ gfp and E. coli (DH5 $\alpha) /$ pET28a-FliC-gfp. M: DNA maker 1 kb; 1: Negative control; 2: $\mathrm{FliC}_{\Delta 220-320}$ gene; 3: $\mathrm{FliC}_{\Delta 220-320^{-}}$ gfp gene; 4: FliC gene; 5: FliC-gfp gene
Expression of recombinant $\mathrm{FliC}_{\Delta 220-320-\mathrm{gfp}}$ and FliC-gfpThe pET28a-FliC $\mathrm{C}_{\Delta 220-320-\mathrm{gfp}}$ and pET28a-FliC-gfp vectors were transformed into E. coli BL21 (DE3) cells. Positive clones were induced by IPTG to produce recombinant proteins. The cultures of both strains after induction showed a green color of GFP, which meant the GFP retained its fluorescent properties when combined with $\mathrm{FliC}_{\triangle 220-320}$ and $\mathrm{FliC}$, facilitating further experiments. To verify the recombinant $\mathrm{FliC}_{\Delta 220-320-\mathrm{gfp}}$ and FliC-gfp proteins, the E. coli cells were lysed, subjected to $12.5 \%$ SDS-PAGE and stained with coomassie blue. The separated bands on gel showed over expression of two proteins of about $73 \mathrm{kDa}$ (lane 2, Fig. 3A) and $82 \mathrm{kDa}$ (lane 2, Fig. 3B), which were exactly the predicted sizes of $\mathrm{FliC}_{\triangle 220-320-\mathrm{gfp}}$ and FliC-gfp, respectively. There was no over expression band in the negative control (lane 1, Fig. 3). In addition, the $\mathrm{FliC}_{\Delta 220-320-\mathrm{gfp}}$ and FliC-gfp were designed to fuse with the His-tag on the C-terminal, so the presence of FliC $\Delta_{220-320-g f p}$ and FliC-gfp were confirmed by using anti-His-tag antibody in Western blot. The results indicated that the proteins excessively

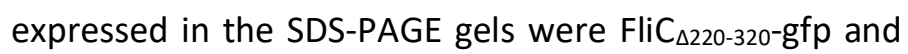
FliC-gfp (lane 3, Fig. 3) and these proteins expressed mainly in the soluble phase. Thus, recombinant $\mathrm{FliC}_{\Delta 220}$ ${ }_{320}$-gfp and FliC-gfp fused to the His-tag were successfully expressed in E. coli BL21 (DE3).
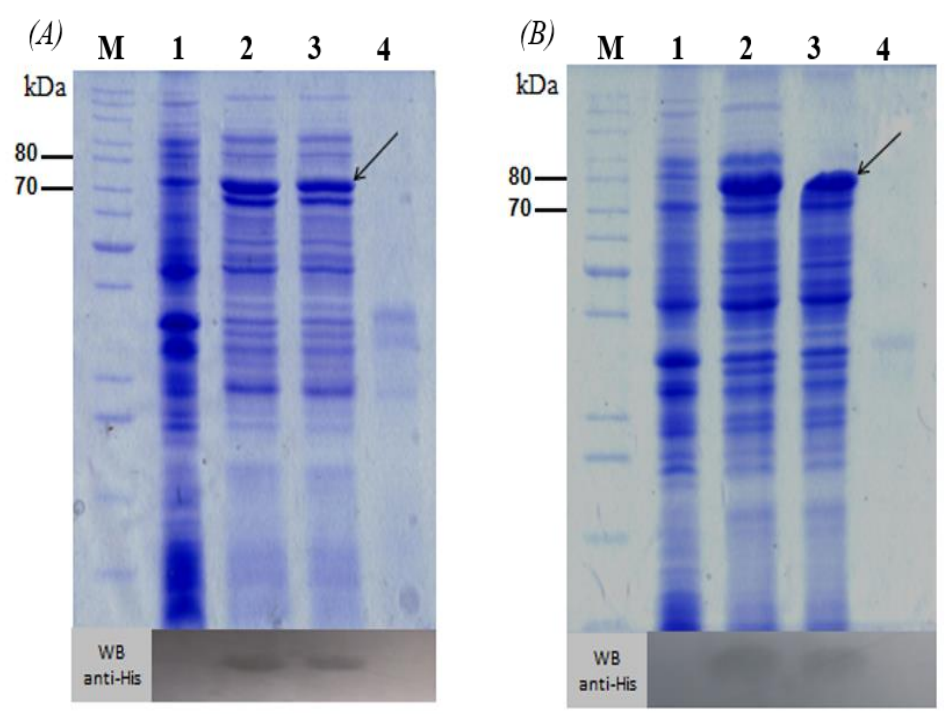

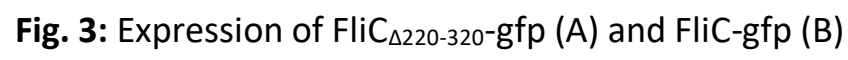
analyzed by SDS-PAGE and Western blot with anti-His-

tag antibody. M: Protein maker; 1 : E. coli BL21 (DE3)/pET28a (+IPTG); 2-4, E. coli BL21 (DE3)/pET28a-

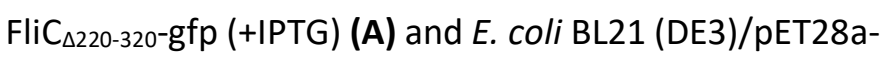
FliC-gfp (+IPTG) (B); 2: Total phase; 3: Soluble phase; 4: Insoluble phase 


\section{Purification of recombinant FliC $\mathrm{C}_{\Delta 220-320-g f p}$ and FliC-gfp-} With the fused His-tag, $\mathrm{FliC}_{\Delta 220-320-\text { gfp }}$ and FliC-gfp were purified using affinity chromatography with $\mathrm{Ni}^{2+}$ column. The proteins those carry the $6 \mathrm{xH}$ is tag were retained through the interaction between the $6 x \mathrm{His}$ and the $\mathrm{Ni}^{2+}$ ions presented in the column. The target proteins were then eluted from the column by the imidazole. Recombinant GFP protein was also expressed, obtained and purified for the evaluation of immune stimulatory of $\mathrm{FliC}_{\Delta 220-320-\mathrm{gfp}}$ and FliC-gfp.

The SDS-PAGE results and the evaluation of purity by Image J software showed the fractions in lane 2, 4 and 6
(Fig. 4A) for a protein band at each lane with the appropriate sizes of FliC-gfp, $\mathrm{FliC}_{\triangle 220-320-\mathrm{gfp}}$ and GFP with a purity of $79 \%, 56 \%$, and $98 \%$, respectively (Figure $4 \mathrm{~B}-$ D). Thus, it was initially purified and successfully acquired the target recombinant proteins to serve for immunological evaluation. However, the recovery

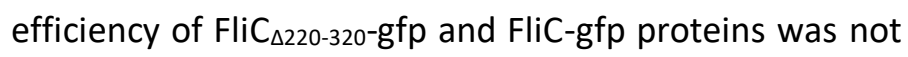
high, compared to GFP. Therefore, further investigation in purification is necessary for the production of a large number of these proteins.

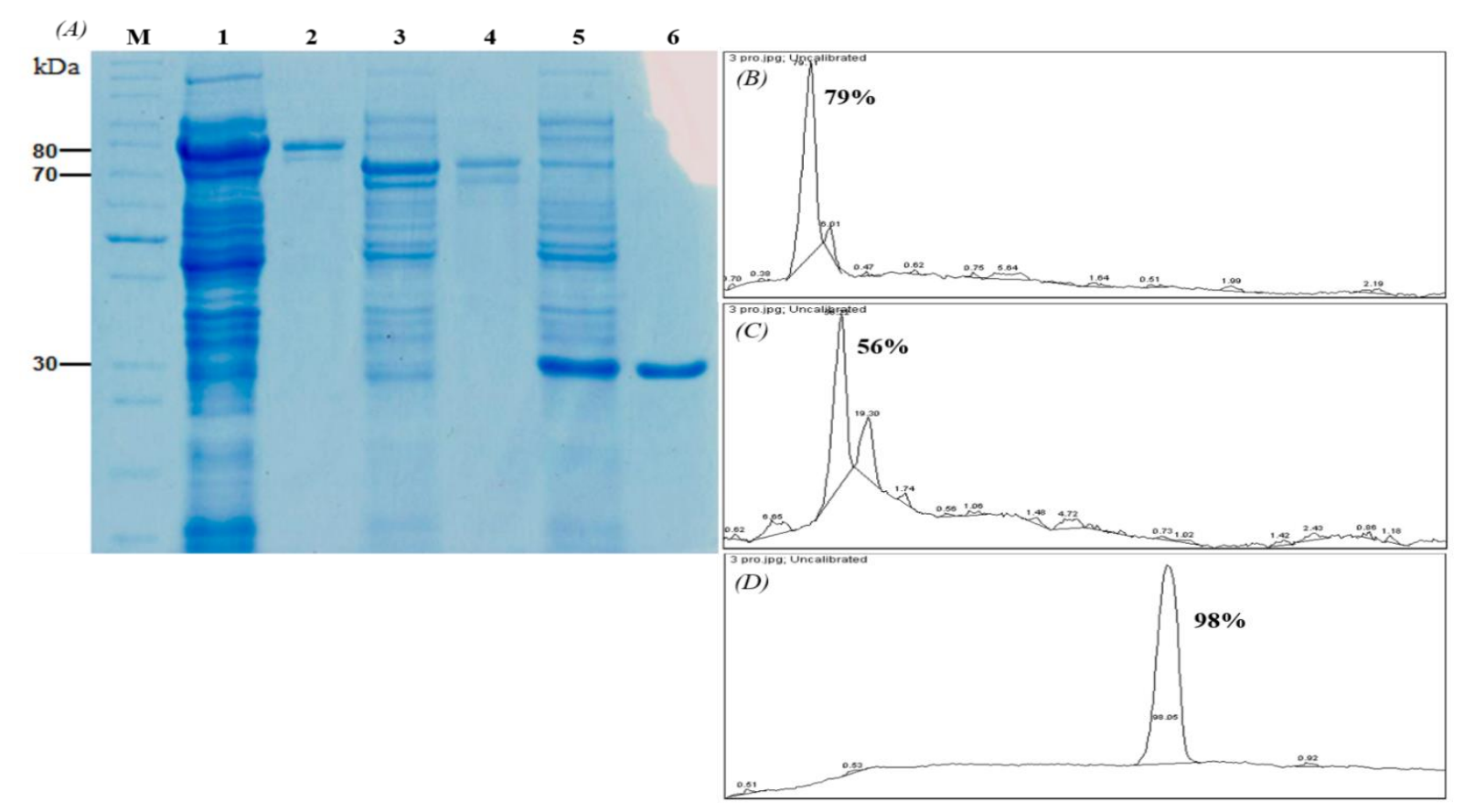

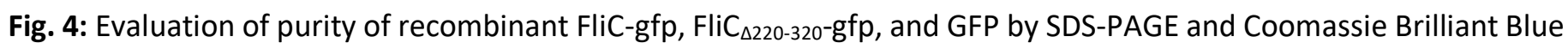

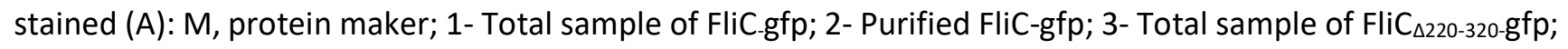

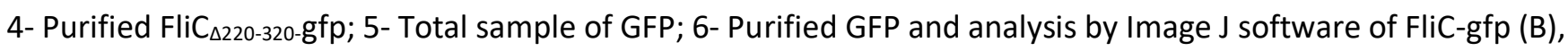

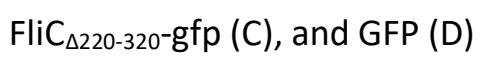

\section{Evaluation of immune stimulatory of FliC $\mathrm{C}_{\Delta 220-320}$ and FliC} in humoral immunity to GFP- To evaluate the ability of $\mathrm{FliC}_{\triangle 220-320}$ and $\mathrm{FliC}$ in humoral immunity stimulation to GFP antigen, GFP specific antibody titers and antigenic characteristic of $\mathrm{FliC}_{\Delta 220-320}$ variances to FliC were determined by using serum samples obtained from experimental mice groups via indirect ELISA.

In case of GFP specific antibody titers, $\mathrm{OD}_{450 \mathrm{~nm}}$ of group 1 $\left(\mathrm{FliC}_{\Delta 220-320-\mathrm{gfp}}+\mathrm{FIA}\right)$ and group 2 (FliC-gfp $\left.+\mathrm{FIA}\right)$ at $1 / 20000$ dilution were 0.602 and 0.799 , respectively, which were 1.3 times and 1.8 times higher compared to the negative control (GFP + FIA) (Fig. 5A). These results suggested that, the combination of GFP protein with $\mathrm{FliC}_{\triangle 220-320}$ and FliC have increased the production of GFPspecific antibodies. The stimulatory effects of $\mathrm{FliC}_{\Delta 220-320}$ and FliC could have come from their abilities to stimulate the innate immune cells' functions, which led to the secretion of pro-inflammatory cytokines activating the adaptive immune system. In particular, when in the fusion form with GFP, $\mathrm{FliC}_{\triangle 220-320}$ and FliC could enhance GFP presentation through the interaction of these two proteins with TLR5 on the surface of antigen-presenting cells. Thus, in the form of fusion with GFP, the $\mathrm{FliC}_{\Delta 220-320}$ variant still can retain the immune stimulatory of the intact Flic. 


\section{A}

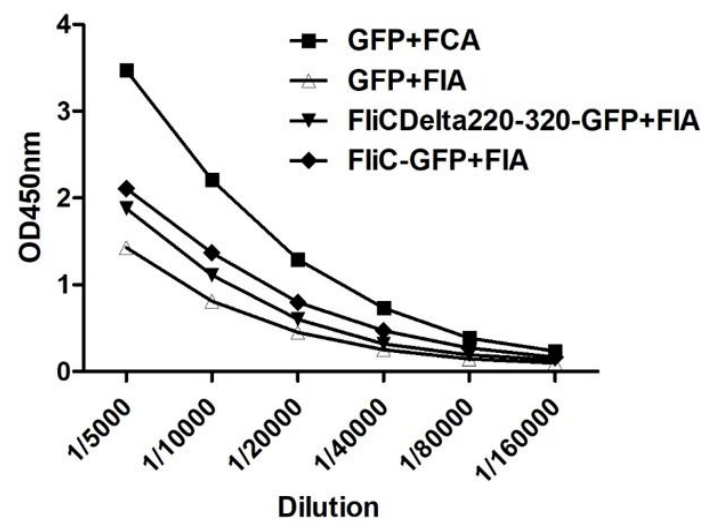

B

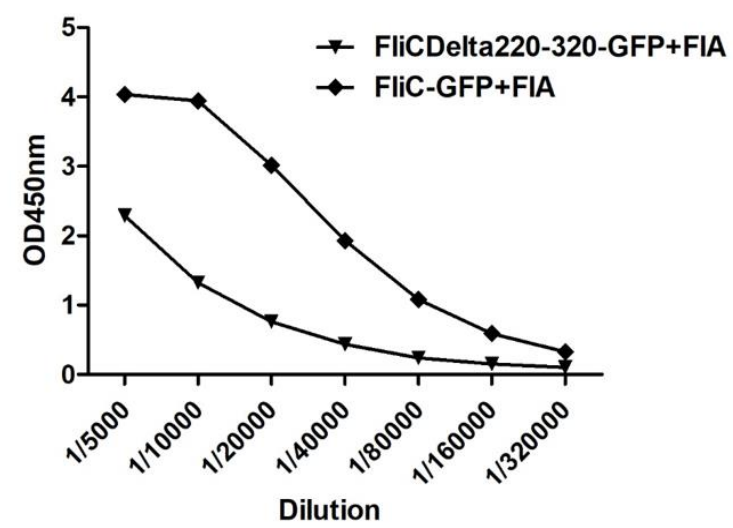

Fig. 5: Evaluation of GFP specific antibody titers (A) and Antigenic characteristic of FliC $\mathrm{C}_{\Delta 220-320}$ variant to FliC (B)

To assess the antigenic characteristic of $\mathrm{FliC}_{\Delta 220-320}$ variant to FliC, the presence of FliC specific antibodies in the antisera sample obtained from group 1 was determined through indirect ELISA. The results showed that the amount of FliC specific antibody of the $\mathrm{FliC}_{\Delta 220-320-\mathrm{gfp}}$ immunized mice were much lower than that of the FliCgfp, specifically, at the $1 / 20000$ dilution, $\mathrm{OD}_{450 \mathrm{~nm}}$ of FliCgfp was 3.013, which was 4 times higher compared to $\mathrm{FliC}_{\Delta 220-320^{-} \mathrm{gfp}}$ (0.761) (Fig. 5B). These results indicated that the removal of 100 amino acids (from 220 to 320 ) in the hypervariable domain of Flic had significantly reduced the antigenic properties of this protein.

\section{DISCUSSION}

Vaccine invention is one of the most crucial and costeffective medical and public health achievements of all time, which helps repel dangerous diseases threating human health. An effective vaccine included a strong immunogen (antigen) and a potent adjuvant (immuno stimulant) that can activate early innate defense mechanisms to aid in the generation of robust and longlasting immune responses ${ }^{[14]}$. Nevertheless, most known adjuvants such as Freund's complete adjuvant (FCA) and Freund's incomplete adjuvant (FIA) are inappropriate for human and animal utilization due to their toxicity and side effects ${ }^{[15]}$. Previous researches indicated that antigenicity of FliC made it potential candidate vaccine adjuvants ${ }^{[6-8]}$. However, its strong antigenicity needs to be eliminated or reduced. In the present study, molecular cloning and recombination expression of $\mathrm{FliC}_{\Delta 220-320-\mathrm{gfp}}$ and FliC-gfp were performed, and immune stimulatory of $\mathrm{FliC}_{\Delta 220-320}$ and $\mathrm{FliC}$ in humoral immunity to GFP were determined to evaluate their adjuvant efficacy. Previous publications of Clement Nempont ${ }^{[16]}$ and Liu et al. ${ }^{[11]}$ on the adjuvant activity of different FliC variants come into the conclusion that immunodominant epitopes, which are responsible for antigenicity of the FliC are in the hypervariable domain. Sequencing and analyzing results from various Salmonella sp. and other bacterial flagellin genes proved that the main antigenic epitopes of flagellin are mainly detected at residues from

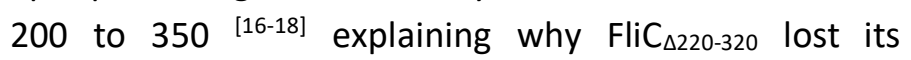
antigenicity but still remained mucosal adjuvancy [11]. However, the $\mathrm{FliC}_{\triangle 220-320}$ variant that Liu et al. ${ }^{[11]}$ created had anti-FliC antibody titers 80 times lower than FliC, compared to $\mathrm{FliC}_{\triangle 220-320}$ variant in fusion with GFP in this study. This dissimilarity might come from the differences between fusion forms with GFP in this study and single forms in Liu's ${ }^{[11]}$ study of the two protein $\mathrm{FliC}_{\Delta 220-320}$ and FliC; or might also be caused by certain test conditions. Additional researches are needed to clarify this. The results suggested that even though the high antigenicity of the adjuvants may be a barrier to its utility for different vaccine applications, but in an immune response activated by $\mathrm{FliC}$ or $\mathrm{FliC}_{\Delta 220-320}$ along with a certain antigen protein, the antigenic properties of the adjuvant neither has a significant correlation with the immune complementary support of these proteins, nor a significant effect to the humoral response towards the target antigen. Further experiments need to be conducted to confirm the ability of stimulating the protective response against antigens of $\mathrm{FliC}_{\Delta 220-320} \mathrm{O}^{\prime} \mathrm{s}$ immune complementation support, and to determine the ability of retaining immunity after being repeatedly exposed. In addition, there have been studies showing that the low antigenicity of $6 x-H i s$ tag causes no affect on the immune response in mice ${ }^{[19,20]}$, so $6 x$-His tag could not interfere with the present data. 


\section{CONCLUSIONS}

The recombinant vectors carrying the genes $\mathrm{FliC}_{\triangle 220-320^{-}}$

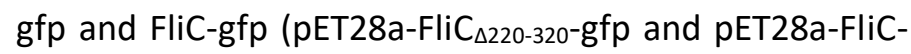
gfp) encoding for $\mathrm{FliC}_{\Delta 220-320-\mathrm{gfp}}$ and FliC-gfp, respectively, had been successfully constructed, in which $\mathrm{FliC}_{\Delta 220-320}$ and $\mathrm{FliC}$ were derived from $S$. enterica Enteritidis. Transformed E. coli BL21 (DE3) with

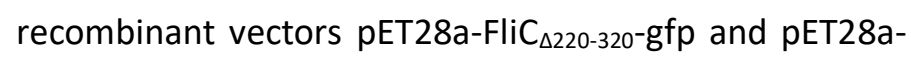
FliC-gfp were successfully created. Expression and purification of recombinant $\mathrm{FliC}_{\Delta 220-320-\mathrm{gfp}}$ and FliC-gfp were performed with a purity of $56 \%$ and $79 \%$, respectively. $\mathrm{FliC}_{\triangle 220-320}$ variant in the fusion form with GFP had four times less antigenicity than FliC (in the FliCgfp fusion) and still retained the FliC's immune stimulation.

Further experiments should be conducted to compare the stimulating effect to the humoral immune response of the mixture of GFP antigen and $\mathrm{FliC}_{\Delta 220-320}$ protein individually with $\mathrm{FliC}_{\Delta 220-320-\mathrm{gfp}}$ fusion protein.

\section{ACKNOWLEDGMENTS}

This research did not receive any specific grant from funding sagencies in the public, commercial, or not-forprofit sectors.

\section{CONTRIBUTION OF AUTHORS}

Research concept- Hieu Tran-Van

Research design- Bao-Chau Thi Tran, Viet-Anh Nguyen, Hieu Tran-Van

\section{Supervision- Hieu Tran-Van}

Materials- Hieu Tran-Van, Thuoc Linh Tran

Data collection- Bao-Chau Thi Tran, Viet-Anh Nguyen, Hieu Tran-Van

Data analysis and Interpretation- Bao-Chau Thi Tran, Viet-Anh Nguyen, Hai-Vy Vo-Nguyen, Hieu Tran-Van

Literature search- Bao-Chau Thi Tran, Hai-Vy Vo-Nguyen, Writing article- Bao-Chau Thi Tran, Hai-Vy Vo-Nguyen, Hieu Tran-Van

Critical review- Hieu Tran-Van

Article editing- Hai-Vy Vo-Nguyen, Hieu Tran-Van

Final approval- Hieu Tran-Van

\section{REFERENCES}

[1] Fauci AS. Infectious diseases: considerations for the $21^{\text {st }}$ century. Clin. Infect. Dis., 2001; 32(5): 675-85.

[2] Leroux-Roels G. Unmet needs in modern vaccinology: Adjuvants to improve the immune response. Vaccine, 2010; 28: C25-C36.
[3] Peggy R, Kai S, Thomas E, Blair P, Carlos AG. Vaccine adjuvants: key tools for innovative vaccine design. Curr. Top. Med. Chem., 2013; 13(20): 2562-80.

[4] Lee S, Nguyen MT. Recent advances of vaccine adjuvants for infectious diseases. Immune network, 2015; 15(2): 51-57.

[5] Guideline on adjuvants in vaccines for human use, Committee for Medicinal Products for Human Use (CHMP), The European Medicines Agency Evaluation of Medicines for Human Use, 2005; pp. 1-18.

[6] Andersen NE, Smith KD, Strobe KL, Barrett SLR, Cookson BT, et al. Evasion of Toll-like receptor 5 by flagellated bacteria. Proce. Natl. Acad. Sci., 2005; 102(26): 9247-52.

[7] Hayashi F, Smith KD, Ozinsky A, Hawn TR, Eugene CY, et al. The innate immune response to bacterial flagellin is mediated by Toll-like receptor 5 . Nature, 2001; 410(6832): 1099.

[8] Boyd S. Design and Production of a Recombinant FliC-Antigen Co-Expression Platform for Increased Vaccine Efficacy (Thesis), 2014; pp. 1-59.

[9] Karam MRA, Oloomi M, Mahdavi M, Habibi $M$, Bouzari S. Vaccination with recombinant FimH fused with flagellin enhances cellular and humoral immunity against urinary tract infection in mice. Vaccine, 2013; 31(8): 1210-16.

[10]Wang G, Shi B, Li T, Zuo T, Wang B, et al. Linear antigenic mapping of flagellin (FliC) from Salmonella enterica serovar Enteritidis with yeast surface expression system. Vet. Microbial., 2016; 184: 20-46.

[11]Liu F, Yang J, Zhang $Y$, Zhou D, Chen $Y$, et al. Recombinant flagellins with partial deletions of the hypervariable domain lose antigenicity but not mucosal adjuvancy. Biochem. Biophys. Res. Commu., 2010; 392(4): 582-87.

[12]Bao-Chau TT, Viet-Anh N, Hieu TV. Cloning, expression and purification of the recombinant FliC from Salmonella enteritidis. Sci. Technol. Dev., 2016; 19: 62-69.

[13]Ausubel F, Brent R, Kingston R, Moore D, Seidman J, et al. Current protocols in Molecular Biology. John. Wiley. Sons, 2003.

[14]Kaur G, Chitradevi S, Nimker C, Bansal A. rlL-22 as an adjuvant enhances the immunogenicity of $\mathrm{rGroEL}$ in mice and its protective efficacy against $S$. Typhi and S. typhimurium. Cellular Mol. Immunol., 2015; 12(1): 96-106. 
[15]Billiau A, Matthys P. Modes of action of Freund's adjuvants in experimental models of autoimmune diseases. J. Leukoc. Biol., 2001; 70(6): 849-60.

[16]Nempont C, Cayet D, Rumbo M, Bompard C, Villeret $V$, et al. Deletion of flagellin's hypervariable region abrogates antibody-mediated neutralization and systemic activation of TLR5-dependent immunity. J. Immunol., 2008; 181(3): 2036-43.

[17]He X-S, Rivkina $M$, Stocker B, and Robinson WS. Hypervariable region IV of Salmonella gene fliCd encodes a dominant surface epitope and a stabilizing factor for functional flagella. Journal of bacteriology, 1994; 176(8): 2406-14.
[18]Kuwajima G. Construction of a minimum-size functional flagellin of Escherichia coli. Journal of bacteriology, 1988; 170(7): 3305-09.

[19] Goldsby RA, Kindt TJ, Osborne BA. Kuby immunology, $4^{\text {th }}$ USA. W, 2000.

[20]Watson DS, Platt VM, Cao L, Venditto VJ, Szoka FC. Antibody response to polyhistidine-tagged peptide and protein antigens attached to liposomes via lipidlinked nitrilotriacetic acid in mice. Clin. Vaccine Immunol., 2011; 18(2): 289-97. 